\title{
Application of Data Mining Technology in Quality Evaluation of Online Teaching Based on 6G
}

\author{
Huan Wang \\ Hubei University of Automotive Technology, China.
}

How to cite this paper: Huan Wang. (2021). Application of Data Mining Technology in Quality Evaluation of Online Teaching Based on 6G. The Educational Review, USA, 5(2), 27-30. DOI: $10.26855 /$ er.2021.02.002

Received: January 29, 2021

Accepted: February 24, 2021

Published: March 1, 2021

Corresponding author: Huan Wang, Hubei University of Automotive Technology, China.

Email: 234885752@qq.com

\begin{abstract}
With the popularization of online teaching, the trend of intelligent education has further increased the needs for communication technology. The Sixth Generation Mobile Communication Standard (Hereinafter referred to as 6G) is becoming an inevitable requirement. With the big data generated by communication technology, we started to thinking about how to make full use of data mining to serve the quality evaluation of online teaching in colleges and universities. In the context of $6 \mathrm{G}$ Communication, this paper puts forward some application scenes of data mining in the subjects and contents of online teaching quality control. Compared with the phased and result-oriented evaluation of traditional quality evaluation, the application of data mining technology based on 6G communication is more student-centered, more objectivity and more effective. For building a high quality education system, it will realize the specific and process-oriented quality evaluation in online teaching of colleges and universities, and achieve continuous improvement.
\end{abstract}

\section{Keywords}

6G, Data Mining, Quality Evaluation, Online Teaching

\section{Introduction}

The rise of online teaching has brought a large number of education data. Thanks to data mining, we can find useful information from the massive chaotic data effectively, which makes work easier and saves time and manpower. From the available literature, the study on the applications of $6 \mathrm{G}$ communication or data mining technology in college education and teaching has already begun (Xu Lei \& Nie Fengying, 2020; Chen Xi, 2020), but this kind of research focuses more on the teaching methods, teaching ideas, and the management of colleges and universities. As to the combination of $6 \mathrm{G}$ communication and data mining applied to the quality evaluation of online teaching, there are very few studies.

The education and teaching quality evaluation system is a complex unit with multiple subjects, multiple levels and multiple objectives. Focusing on the system and using $6 \mathrm{G}$ communication technology, this paper constructs an evaluation database of students, teachers, colleagues, supervisors and also administrators, and sets up a systematic platform to realize instant, accurate and complete sample monitoring. Thus, supplemented by data mining technology, we can carry out correlation, classification and clustering analysis of the data generated in education and teaching quality monitoring and evaluation system, and finding the existing problems. For education and teaching quality management, accurate and predictable information can be provided and continuous improvement can be realized.

\section{Data Mining Based on $6 \mathrm{G}$}

Data mining is a process of finding required and corresponding data information from big data. Its main capabilities are data cleaning, data integration, data selection, data transformation, pattern discovery, pattern evaluation, knowledge representation, etc. 
Nowadays, the global scientific and technological revolution and industrial transformation are accelerating. The wide application of the new generation of information and communication technologies, such as artificial intelligence (AI), virtual reality (VR), augmented reality (AR), 3 Dimensions (3D) media, Internet of Things (IoT), etc., have produced huge transmission data. As a result, the rapid growth of mobile data traffic puts forward higher requirements for the iteration of mobile communication system, which means the arrival of the $6 \mathrm{G}$ era.

On November 3, 2019, China set up the National 6G Technology R \& D Promotion Working Group and the Overall Expert Group, marking it the official launch of China's 6G research and development. Not coincidentally, Huawei's 6G development has been promoted in parallel with 5-Generation (5G). As for the operators, China Telecom, China Mobile and China Unicom have all started the research and development of $6 \mathrm{G}$.

Based on 5G vision, 6G will be further expanded and upgraded (Cui Chun-feng, Wang Sen, Li Ke, Dong Jing \& Zheng Zhi-min, 2020). 5G has brought the connection between people, as well as people and things to reality. 6G will complete the information interaction between things and accomplish a network where everything is connected, that is the realization of IoT. 5G proposed three application scenarios, including large bandwidth, massive connection, and ultra-low latency. On this basis, 6G will continuously improve its performance and optimize experience through technological innovation, and further extend the boundaries of services from the physical world to the virtual world.

In the field of education, intelligence is becoming an irreversible trend. With the increasing demand for online teaching, $5 \mathrm{G}$ is providing new functions and better quality of service (QoS) undoubtedly. Modern education combined with VR/AR presents a new teaching experience, which is more interactive, personalized and immersive for teachers and students. It can greatly enhance students' interest in learning and the rapid absorption of knowledge.

However, the rapid growth of data-centric intelligent systems poses great challenges to the capability of 5G wireless systems. To overcome the performance limitation of $5 \mathrm{G}$ and to realize the vision of intelligent education, the applications must be supported by wireless high-speed communication with reliable connectivity. And the generation of 6G IoT communication technology provides a strong support for the further expansion of data mining. The 6G wireless system can break through the unified network of ubiquitous intelligence between the virtual and real space. In the future intelligence education, as one of the most important links, education and teaching quality evaluation system needs to dig deep into the massive teaching data, which cannot be separated from the support of 6G IoT communication technology.

\section{Applications of Data Mining in Quality Evaluation}

As the main battlefield of $6 \mathrm{G}$ intelligence education, the holographic communication and holographic display brought by intelligent wearable devices and extended reality (XR) will make a difference in online teaching. With the emergence of big data, data mining of online teaching quality can realize the correlation analysis of the whole sample, which means dynamic adjustment and continuous improvement in quality control. Therefore, the education and teaching quality evaluation of colleges and universities is in desperate need of data mining.

\subsection{The Subject of Quality Evaluation}

(1) For students, real-time evaluation can be realized.

When it comes to students' teaching evaluation, most colleges and universities choose to set up some periodical evaluation time node for students to evaluate the teaching effect, such as mid-term or final. Obviously, this kind of time point lacks pertinence. For a short course, it may end well before the final comes. When students can evaluate the teacher or the course, the teaching impression has already faded and the effect can be imagined.

With the help of 6G IoT communication technology, real-time evaluation can be realized. No matter in class or after class, students can monitor and evaluate the teaching quality at any time through the support of data mining algorithm. Compared with the previous periodical evaluation, the real-time evaluation is more targeted and effective for improving the quality of education and teaching

(2) For teachers, accurate self-evaluation can be realized.

Currently, teachers' self-evaluations are more subjective. The tendency of overestimating or underestimating in self-evaluation is inevitable. In the $6 \mathrm{G}$ era, class data can be collected through intelligent devices, such as intelligent wearable devices, sensors and cameras. The teaching situation will be analyzed by data mining and artificial intelligence teaching system. Meanwhile, some typical performance can be identified and extracted, such as the change of class activity and students' attention. After class, the teacher can look back these videos and analyze the teaching effects. In this way, teachers' self-evaluation can be more objective and accurate.

(3) For other subjects, remote- evaluation can be realized.

With the support of 6G IoT communication technology, holographic communication and holographic display brought by XR can realize multi-point remote interactive teaching. Teaching scenes can be tracked, identified and switched 
automatically. The main classroom screen can automatically switch between the panoramas of classroom and students, the close-up of teacher and students, the blackboard and courseware.

So for other subjects of evaluation, such as secondary colleges, supervisors, administrative departments, and even parents and the public, can monitor the teaching process remotely through $6 \mathrm{G}$ network and XR. With the opening of appropriate authority, the instant remote-evaluation can be realized and the classroom situation can be monitored at any time. By making individualized teaching evaluation scale, teaching evaluation schedule and real-time evaluation table, the real-time statistical data will be fed back to do data mining. Then the quality of education and teaching in colleges and universities will be more efficient in monitoring and evaluation.

Furthermore, data mining can output multi-dimensional and multi-level reports for all kinds of evaluation subjects, such as the teachers, students, and other subjects. And data mining can also provide high coverage, personalized and customizable data analysis dimensions, so as to meet the analysis needs of different universities, colleges, majors and courses.

\subsection{The Content of Quality Evaluation}

(1) Pre-class Preparation and Teaching Materials

With the support of $6 \mathrm{G}$ IoT technology, PC, mobile phones, intelligent wearable devices and other intelligent devices can be connected with each other, and as well as the professional teaching resource database. Both teachers and students can easily access to the teaching resource database, wherever and whenever they need.

Data mining technology can not only reduce the tedious preparation of paper materials, but also further improve the quality of the screening, collection and updating of teaching materials. It will make the work more efficient and help teachers find better teaching resources from the huge amounts of data, which may have higher relevancy and better quality.

Furthermore, the evaluation of teaching preparation will not be limited to the presence or absence of the presented teaching materials, but can be monitored and checked in the process of teaching preparation through data mining technology. That means further examination of the pre-class preparation quality and the implementation of pre-teaching process assessment.

(2) The Attendance of Teachers and Students

At present, in higher education, class attendance has always been a problem for teachers. In a traditional way, a teacher usually checks the attendance by roll call. But when it comes to the large classes, such as public subjects, the roll call is extremely time-consuming. And there may also be situations that students answer for others. In the current $5 \mathrm{G}$ environment, although various online teaching tools have been produced and a variety of check-in methods have been provided, like sign in with photo, location or gesture, the above problems are still not been fundamentally solved.

Unlike the IoT era, the traditional teaching equipment is relatively independent. Now the intelligent teaching devices can be integrated into an interconnected network through the IoT, which can greatly improve the utilization rate of teaching equipment. So the intelligent management of attendance can be realized by automatically identifying and checking the identity of students or teachers. It can tell whether the teacher arrives at class on time or leaves early. And the students' attendance or early withdrawal does not need teacher's roll call or students' check-in. The intelligent automatic identification will not only improve students' attendance rate, but also become a great class time saver.

(3) The Recognition and Analysis of Classroom Behavior

With the arrival of $6 \mathrm{G}$ technology and the further maturity of the interdisciplinary disciplines, such as biological science, material science, bio-electronic medicine, etc., it is expected to realize the complete "human digital twinning" in the future (Liu et al., 2020). A large number of intelligent sensors will be applied to the human body. And an accurate and real-time "mirror mapping" of important organs, respiratory system, nervous system, emotional state, urinary system, musculoskeletal, etc., will be formed. Then there will be an accurate replica of the virtual world of the whole human body, and realize the real-time monitoring of human personalized behavior data. Based on big data and artificial intelligence, $6 \mathrm{G}$ artificial intelligence education is definitely the brain of intelligent education. It can achieve intelligent analysis and visual management of classroom, learning, sports and teaching behavior, and also achieve better guidance and promotion of intelligent teaching.

From all kinds of data collected by intelligent wearable devices, sensors, cameras and other intelligent equipment, human behavior recognition technology can be used to detect the combination and movement of facial expressions, head, neck, shoulder, elbow, hand, hip, knee, foot and other key points of human skeleton (Zhang Wenmei, Qi Binbin, \& Fan Wenxiang, 2021). And students' classroom behavior and emotional changes can also be identified and analyzed by standing, raising hands, sitting up, turning sideways, leaning over the table and other behavior. Based on the feedback data, we can analyzes students' concentration, activity and typical emotion, and finally help teachers to 
understand the key active links in class, the distribution of students' active areas, the proportion of classroom emotion and behavior. The statistical data will be displayed through a visual image, so the trend of classroom behavior and the change of students' emotion and learning attitude can be seen clearly. Teachers can see how their contents appeal to their students and pay attention to the learning state of each student as a reference. Then the teachers can adjust their teaching progress and methods, and improve teaching effectiveness, which on the contrary will help the school to carry out more detailed teaching evaluation and more reasonable teaching management.

Besides, the 6G IoT technology can also be used for automatic speech recognition (ASR) to collect the data of interactions between teachers and students in class. First, the artificial intelligence records will transform the students' speeches and teacher's teaching contents into text. Then, through text technology, the unstructured data is transformed into structured data. Through the change of the classroom atmosphere, the key words of interaction will be automatically marked and the positive words which are conducive to the classroom atmosphere will be extracted. The positive vocabulary for students' learning enthusiasm can also be extracted to help teachers and parents improve the effect of teaching interaction and learning efficiency. For example, at present, many teachers are exploring and implementing ideological and political theories teaching in all courses. The ASR can be used to evaluate the teaching quality. By keywords extraction, supervisors can truly understand the situation whether or not the teacher has carried out ideological and political theories teaching. And the implementation effect and students' responses can be known as well.

(4) The Management and Improvement of Teaching Quality

Based on the data mining technology, we can achieve total quality management both online and offline through the adjoint data acquisition and dynamic evaluation analysis. For each teacher and student, the evaluation results, academic reports and personalized intelligent promotion plan can be provided. According to the different situation of each student, we can offer targeted learning resources and knowledge points. Then teachers can teach students in accordance of their aptitude, and the administrators can reach overall supervision and get decision-making assistance. As for each teacher or each course, multi-dimensional teaching report and teaching file can be formed. By analyzing historical data, administrators can understand whether teachers have met the requirements in the teaching process, such as continuous improvement of teaching quality, updating of teaching methods and contents, etc.

\section{Summary}

Based on 6G communication technology and making full use of data mining, the quality evaluation of online teaching in colleges and universities can achieve the remote real-time evaluation with multi-subject, multi-dimensional and full sample. Teaching evaluation is not only the evaluation of teachers' teaching quality, but also the evaluation of students' learning conditions. Supported by 6G communication technology, the obtained data are more objective, reliable and undelayed. Through data mining technology, both teachers and students can get objective judgment and clearer self-cognition. For teachers, we can immediately understand the feedback of students, and dynamically improve the quality of teaching. For students, they can feedback the quality of teaching anytime and anywhere, and further improve the evaluation quality of the education and teaching in colleges and universities.

\section{Acknowledgements}

This work was supported by 2020 Logistics Education Reform and Research Project Plan (No. JZW2020216) and 2020 Teaching Research and Reform Project of Hubei University of Automotive Technology (No. JY2020036).

\section{References}

Chen Xi. (2020). Research on the Development Path of Data-Driven Teaching Reform in Universities [J]. Theory and Practice of Education, 2020, 40(33): 52-55.

Cui Chun-feng, Wang Sen, Li Ke, Dong Jing, Zheng Zhi-min. (n.d.). 6G Vision, Scenarios and Network Requirements [J/OL]. Journal of Beijing University of Posts and Telecommunications: 1-8. [2021-01-25]. http://ras.huat.edu.cn:80/rwt/CNKI/https/ MSYXTLUQPJUB/10.13190/j.jbupt.2020-160.

Liu Guangyi, Jin Jing, Wagn Qixing, et al. (2020). Vision and Requirements of 6G: Digital Twin and Ubiquitous Intelligence [J]. Mobile Communications, 2020, 44(06): 3-9.

Xu Lei, Nie Fengying. (2020). The Research Status and Technical Paths of the Educational Big Data Applied in Educational Management [J]. Jiangsu Higher Education, 2020(12): 69-73.

Zhang Wenmei, Qi Binbin, Fan Wenxiang. (2021). Analysis of Data-driven Teaching Behavior: Current Situation, Basic Logic and Development Trend [J]. Journal of Distance Education, 2021, 39(01): 84-93. 